\title{
The effect of spatial attention on invisible stimuli
}

\author{
KILHO SHIN \\ Yonsei University, Seoul, Korea \\ Moritz Stolte \\ Birkbeck College, London, England \\ AND \\ SAng Chul Chong \\ Yonsei University, Seoul, Korea
}

\begin{abstract}
The influence of selective attention on visual processing is widespread. Recent studies have demonstrated that spatial attention can affect processing of invisible stimuli. However, it has been suggested that this effect is limited to low-level features, such as line orientations. The present experiments investigated whether spatial attention can influence both low-level (contrast threshold) and high-level (gender discrimination) adaptation, using the same method of attentional modulation for both types of stimuli. We found that spatial attention was able to increase the amount of adaptation to low- as well as to high-level invisible stimuli. These results suggest that attention can influence perceptual processes independent of visual awareness.
\end{abstract}

It is a well-established fact that visual information, after entering through our eyes, is processed in a hierarchical fashion at multiple stages of the visual system. Early stages encode low-level features, such as edge orientation and contrast, whereas complex stimuli, such as faces, are represented at later stages (Marr, 1982). This processing hierarchy provides the backdrop for the investigation of unconscious visual processes. By measuring the psychophysical effect elicited by a visual stimulus not consciously perceived, it may be possible to determine whether or not, at a certain stage, awareness is required for visual processing. Furthermore, attention is known to influence processing at multiple levels of the hierarchy (Kastner \& Ungerleider, 2000). Interestingly, recent studies have suggested a dissociation between attention and conscious perception, on the basis of the finding that attention is able to modulate early visual processes in the absence of awareness (Bahrami, Carmel, Walsh, Rees, \& Lavie, 2008; Blake, Tadin, Sobel, Raissian, \& Chong, 2006; He, Cavanagh, \& Intriligator, 1996; Kanai, Tsuchiya, \& Verstraten, 2006). Most of these studies exploited the fact that an image presented to one eye becomes temporarily invisible when an incompatible image is presented to the other eye (binocular rivalry). Moreover, by dynamically changing the stimulus in one eye, the competing stimulus can be suppressed from awareness for long periods of time. The dependent measure is usually a visual aftereffect modulated by adaptation to visible as well as to invisible low-level stimuli. For example, Bahrami et al. showed that spatial attention modulates the tilt aftereffect, even without being aware of the stimulus. The same effect has been demonstrated for feature-based attention (Kanai et al., 2006).

So far, only a few studies have investigated the effects of attention on unconscious processing of complex, highlevel stimuli. Finkbeiner and Palermo (2009) demonstrated that faces were processed regardless of attention to an invisible prime, whereas nonface stimuli required spatial attention in order to be processed unconsciously. Furthermore, Moradi, Koch, and Shimojo (2005) have claimed that conscious perception of a face stimulus is required to produce the identity-specific aftereffect.

Here it is important to note that, in contrast to the findings discussed above, there is also evidence that suggests a larger effect of spatial attention at later, rather than earlier, stages of processing (Kastner \& Ungerleider, 2000; Reynolds \& Desimone, 1999). Moreover, according to reverse hierarchy theory, attention begins to exert its effects at higher levels of processing (Hochstein \& Ahissar, 2002), suggesting that attentional modulation should be stronger for high-level stimuli. The contradictory findings may in part be explained by the differences in methodology, specifically in the control of attentional engagement. In fact, by using low-contrast stimuli adaptors, Bahrami et al. (2008) were able to demonstrate an effect of spatial attention previously not observed with high-contrast adaptors (e.g., Kanai et al., 2006).

In the present experiments, we show that spatial attention to invisible gratings produces significant elevations in contrast threshold. Furthermore, spatial attention to

S. C. Chong, scchong@yonsei.ac.kr 
invisible faces produced a robust gender aftereffect. The amount of attentional capture at the attended location was controlled by a contrast-decrement detection task that was equated in terms of difficulty for all participants. Our results suggest that the unconscious processing of both low-level and high-level stimuli is influenced by spatial attention.

\section{EXPERIMENT 1}

The purpose of Experiment 1 was to test whether endogenous spatial attention can influence adaptation to invisible gratings. The paradigm we adopted was designed to overcome methodological differences of previous studies and to refine our understanding of the conditions required for spatial attention to affect the processing of lowlevel invisible stimuli. In accordance with the findings of Bahrami et al. (2008), we used an attentionally demanding task to focus attention on a specific spatial location. The threshold elevation aftereffect (TEA) was used to estimate the attentional effect on adaptation. In order to control for attentional bias, we measured the TEA in the test phase at random locations, independent of the previously attended location during the adaptation phase.

\section{Method}

Participants. Five healthy volunteers, including the first author, participated in Experiment 1. Except the author, all participants were naive to the purpose of the experiment. All participants had normal color vision and normal or corrected-to-normal visual acuity and right-eye dominance, determined by pointing at a distant object. Every aspect of this study was carried out in accordance with the regulations of the Departmental Review Committee of Yonsei University.

Apparatus and Stimuli. The stimuli were created using the Psychophysics Toolbox (Brainard, 1997; Pelli, 1997) and presented on a linearized Samsung 21-in. monitor driven by a Pentium VI computer. The frame rate of the monitor was $85 \mathrm{~Hz}$. The participants were positioned approximately $90 \mathrm{~cm}$ from the display, with their heads held in chin- and forehead rests. At this distance, a pixel subtended approximately $0.0155^{\circ}$ of visual angle. Participants viewed the left and right halves of the screen through a mirror stereoscope. A small fixation cross and nonius lines were always present at the center of the screen to facilitate binocular alignment.

Adaptors were sinusoidal gratings, rendered invisible by interocular suppression. To completely suppress the adaptors, dynamic radial gratings were used as suppressors, alternating between clockwise and counterclockwise rotation at $1 \mathrm{~Hz}$. These suppressors do not produce motion- or orientation-specific aftereffects (Blake et al., 2006). Two sinusoidal gratings (adaptors) were presented to the left and the right visual field of the nondominant eye, and two radial gratings (suppressors) were presented to the left and the right visual fields of the dominant eye. The size of adaptors and suppressors was $0.93^{\circ}$, effectively minimizing the occurrence of mixed percepts (Blake, 2001). Adaptors were flickered at $4 \mathrm{~Hz}$ to prevent afterimage formation. Their orientation was either $45^{\circ}$ or $135^{\circ}$, with a spatial frequency of $8.8 \mathrm{cpd}$. The contrast of the suppressors was always $100 \%$, whereas the contrast of the adaptors ( $47 \%$ to $74 \%$ ) varied, depending on individual contrast thresholds. Each stimulus was surrounded by a high-contrast checkerboard frame to maintain binocular fusion. Using the bit-stealing technique (Tyler, 1997), we created 1,792 luminance steps to measure contrast thresholds. In the test phase, the stimuli were the same gratings used as adaptors, except that contrast was varied, depending on the participants' performance.
Procedure. The experimental procedure consisted of four phases: (1) contrast-threshold estimation, (2) contrast-decrement threshold estimation, (3) adaptation, and (4) testing. In the first phase, baseline contrast thresholds were estimated to be used in the adaptation and testing phases. In the second phase, the amount of contrast reduction in the suppressors required to reach a change detection criterion of $75 \%$ was determined for each participant to equate attentional demands during the adaptation phase. Subsequently, in the adaptation phase, participants detected contrast decrements in one of the two suppressors while adapting to gratings suppressed from awareness. In the testing phase, postadaptation contrast thresholds were estimated and the attentional effect was defined as the difference between pre- and postadaptation thresholds: the threshold elevation index (TEI)

Contrast-threshold estimation. Participants' contrast thresholds for the sinusoidal adaptor gratings were measured using interleaved QUEST staircases (Watson \& Pelli, 1983). We used four staircases (two for each side of the visual field) for each of the two possible grating orientations $\left(45^{\circ}\right.$ and $\left.135^{\circ}\right)$. The estimated thresholds were averaged and served as the preadaptation baseline.

At the beginning of every trial, a fixation point, a nonius line, and two checkerboard frames were presented to each eye (Figure 1A). Upon a keypress, one of the two checkerboard frames flickered twice for $200 \mathrm{msec}$ with a 200 -msec interval to indicate the location of threshold measurement. Subsequently, a grating was displayed for $250 \mathrm{msec}$ in the cued location, either directly after the flicker (first interval) or after a delay of $300 \mathrm{msec}$ (second interval). Participants were asked to indicate during which of the two intervals a grating had been presented and whether they were confident in or uncertain of their decision. If participants thought a grating had been presented in the first interval, they pressed " 4 " if confident, or " 1 " if uncertain. If participants thought a grating had been presented in the second interval, they pressed " 5 " if confident, or " 2 " if uncertain. If the response was incorrect, they heard a short, low-pitched tone. To facilitate convergence of the QUEST staircases, participants could indicate how confident they were in their response by pressing either "4" or " 5 ." A confident response, if correct, was treated as three correct keypresses. To encourage the careful use of such keys, the session was terminated if a participant indicated confidence in an incorrect response; in this case, the participant had to redo the terminated session.

Adaptation and attentional task. During the adaptation phase (Figure 1B), participants tracked reductions in contrast for the suppressor at the attended location. To equate attentional demands between participants, the amount of contrast reduction required to be correctly detected in $75 \%$ of the trials was estimated using a staircase procedure (Levitt, 1970). When participants were correct for two successive times, the contrast decrement of the suppressor was decreased by $4 \%$, but when they were incorrect, it was increased by $4 \%$. Each block ended after 17 reversals and we defined the threshold for contrast-decrement detection as the average of the last 4 reversals. Adaptors were always presented to the nondominant eye with a contrast of $16 \times$ baseline threshold, but suppressors were only presented to the dominant eye at $100 \%$ original contrast. The procedure was repeated four times, once for each adaptor orientation and locus of attention ( $45^{\circ} / \mathrm{left}, 135^{\circ} / \mathrm{left}, 45^{\circ} /$ right, and $135^{\circ} /$ right; order counterbalanced across participants). The average of the four values was used for the attentional task in the adaptation phase, ranging from $33 \%$ to $67 \%$ contrast decrement.

At the beginning of every trial, two fixation points, two nonius lines, two adaptors, and two suppressors surrounded by checkerboard frames were presented on the display, and the location of the suppressor for the attention task was indicated. For $60 \mathrm{sec}$, participants adapted and detected contrast decrements for the suppressor at the attended location. The suppressor was presented at decreased contrast for $110 \mathrm{msec}$ and participants were allowed $1 \mathrm{sec}$ to respond by pressing "A." If a decision was incorrect, a short, low-pitched tone was heard. Additionally, participants were asked to track their 
A

B Nondominant eye Dominant eye

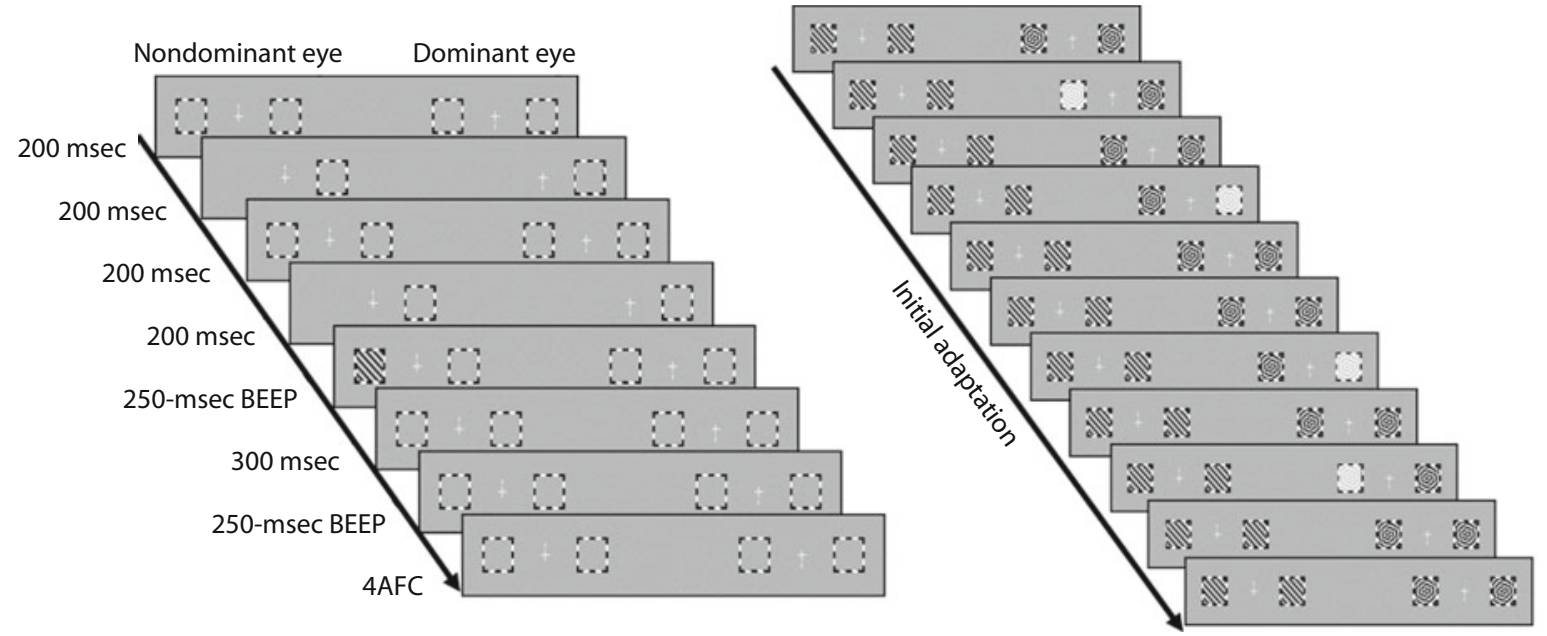

Figure 1. Schematic diagram of the procedure of Experiment 1. Two checkerboard frames, one fixation point, and one nonius line were presented to each eye. There was a 60-sec initial adaptation period with two dynamic radial gratings (suppressors) presented to the dominant eye and two sinusoidal gratings (adaptors) presented to the nondominant eye. A test stimulus that was the same as the adaptor was presented either to the left or to the right of the fixation point in one of the two intervals, after one of the two checkerboard frames was flickered twice. Participants were asked to make a four-alternative forced choice (4AFC) to indicate the interval in which a grating was presented and their confidence level ("certain" or "uncertain"), followed by a 5-sec top-up adaptation. During both adaptation periods, participants performed the contrast-decrement detection task on one of the suppressors and tracked their percept of the suppressor or adaptor at the same time. The procedure ended after 80 trials. (A) Procedure for contrast-threshold estimation. (B) Procedure for the contrast-decrement detection session.

percepts. They held down "2" while perceiving the suppressor and " 1 " while perceiving the adaptor. The results showed that the adaptors were successfully suppressed from awareness $97.2 \%$ to $99.5 \%$ of the time. After the adaptation phase, participants performed a single trial of postadaptation contrast-threshold measurement at a randomly chosen location (left or right), followed by $5 \mathrm{sec}$ of top-up adaptation.

The procedure in the main experiment was the same, except that the number of trials was fixed at 80 per block and the previously measured contrast-decrement detection threshold was used throughout. Participants performed the detection task during each initial adaptation $(60 \mathrm{sec})$ and top-up adaptation $(5 \mathrm{sec})$ period. Postadaptation contrast measurements, with exactly the same procedure as the baseline-contrast threshold measurements (except that now the number of trials was fixed), were interleaved with top-up adaptation periods. We did not use different orientations for the test stimuli, since a previous study with a similar method had shown this aftereffect was orientation specific (Blake et al., 2006).

Suppressed and yoked sessions in the main experiment. In the main experiment, we manipulated two independent variables in a within-participant design: (1) locus of attention (left or right), and (2) orientation of the adaptor $\left(45^{\circ}\right.$ or $\left.135^{\circ}\right)$. There were two sessions with four experimental blocks each ( 2 loci of attention $\times 2$ adaptor orientations). In the first session, the adaptors and suppressors were both presented simultaneously. However, in the second session, presentation of adaptors and suppressors alternated according to the reported percept in the previous session. In this way, we were able to rule out the possibility that the effect of adaptation was merely due to the presence of the suppressors.

\section{Results and Discussion}

The average contrast threshold before adaptation was $3.64 \%$. The amount of contrast reduction needed to perform at $75 \%$ correct in the attention task ranged from $33 \%$ to $67 \%$. Figure 2 shows the main results. The TEI was defined as the ratio between contrast thresholds before and after adaptation (postadaptation threshold divided by preadaptation threshold). Note that a TEI value above 1 indicates an effect of adaptation. One-sample $t$ tests indicated that the TEIs of the suppressed session were significantly different from 1 [attended field, $t(4)=6.372, p<.05$; unattended field, $t(4)=3.590, p<.05]$, suggesting that the adaptors induced significant adaptation even when suppressed from awareness. However, TEIs of the yoked session were not significantly different from 1 [attended field, $t(4)=1.705, p=.163$; unattended field, $t(4)=$ $1.104, p=.332$ ], suggesting that the mere presence of the suppressors was not enough to evoke adaptation. Since participants perceived the adaptors for only about $2 \%$ of the time, this result was expected.

Furthermore, we found an effect of endogenous spatial attention on the amount of adaptation in the suppressed session. Paired-sample $t$ tests indicated that the TEI was significantly higher when the grating was presented at the attended location (2.06) rather than at the unattended location $[1.17 ; t(4)=9.718, p<.01]$, suggesting that endogenous spatial attention modulated the amount of adaptation to invisible gratings. These results are in line with the findings of Bahrami et al. (2008) and support the claim that both an attentionally demanding task and lowcontrast adaptors are required to focus spatial attention sufficiently.

\section{EXPERIMENT 2}

The purpose of Experiment 2 was to test whether endogenous spatial attention can influence the processing of complex stimuli without visual awareness. More specifi- 


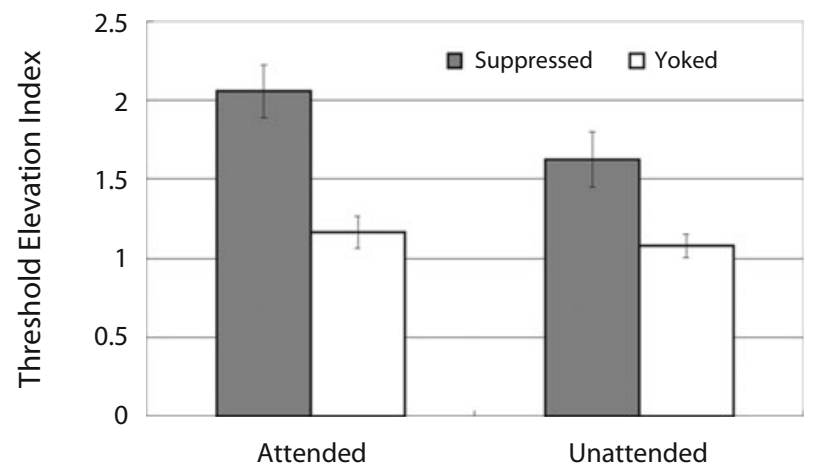

Figure 2. Results of Experiment 1. Threshold elevation index for suppressed condition (gray bar) and yoked condition (white bar). Error bars represent standard errors of the means.

cally, we investigated whether the gender aftereffect for faces, which is presumably processed in higher stages of the visual hierarchy, can be modulated by spatial attention in the absence of conscious perception.

Although recent findings suggest that face processing is neither modulated by spatial attention to a suppressed stimulus (Moradi et al., 2005), nor by temporally focusing attention on an invisible cue in a priming paradigm (Finkbeiner \& Palermo, 2009), the lack of behavioral effects does not preclude the possibility of neural modulation in face-selective areas. Indeed, neural activity in the right fusiform face area (FFA), although reduced for invisible as opposed to consciously perceived faces, was still measurable (Jiang \& He, 2006). Furthermore, the processing of emotional faces has been shown to be modulated by attentional load (Pessoa, McKenna, Gutierrez, \& Ungerleider, 2002).

Lastly, using the same paradigm, including the same attentional task, to measure adaptation to invisible low-level stimuli, such as gratings, as well as to high-level stimuli, such as faces, will allow us to draw firm conclusions regarding the influence of attention at different stages of the visual processing hierarchy.

\section{Method}

Participants. Seven healthy volunteers participated in Experiment 2. All were naive to the purpose of the experiment and had normal or corrected-to-normal vision. Four of them were right-eye dominant. Dominance was determined by pointing at a distant object. Every aspect of this study was carried out in accordance with the regulations of the Departmental Review Committee of Yonsei University.

Apparatus and Stimuli. The apparatus was the same as in Experiment 1 . Four neutral face images (two male and two female) were chosen from a face database (Chung, Oh, Lee, \& Byun, 1998). All pictures were frontal views without hair, grayscale, and equal in size $\left(2.64^{\circ} \times 2.64^{\circ}\right)$. Two sets of five pictures were produced by morphing a male with a female face with Fantamorph software (Abrosoft Fantamorph Version 3.0) (male-female ratio: 94\%-6\%, $72 \%-28 \%, 50 \%-50 \%, 28 \%-72 \%, 6 \%-94 \%$; Figure 3). A mask was created from a random dot pattern composed of equal density black-and-white squares $\left(0.2^{\circ} \times 0.2^{\circ}\right)$ having the same size as the morphed faces.

Adaptors were $100 \%$ female faces. To suppress them, the same dynamic radial gratings as in Experiment 1 were presented to the left and the right visual field of the nondominant eye, whereas the two adaptors were presented to the left and the right visual fields of the dominant eye. Each stimulus was surrounded by a high-contrast checkerboard frame to maintain stable binocular alignment. The contrast of the suppressor was always $100 \%$, and the contrast of the adaptor was $50 \%$.

Procedure. We obtained points of subjective equality (PSEs) of male-female discrimination both before and after adaptation. We defined the amount of adaptation as the ratio between the pre- and postadaptation PSE. During adaptation, participants performed the contrast-decrement detection task on the attended suppressor, as in Experiment 1

Baseline PSE estimation. To measure PSEs of male-female discrimination, participants had to decide whether a morphed face looked male or female. The session consisted of two blocks. In the first block, morphed faces from the first set were presented to the right visual field of the nondominant eye, and morphed faces of the second set were presented to the left visual field of the nondominant eye. In the second block, assignments to the visual fields were reversed for the two sets. Note that we used two different locations to present two sets of faces, and that these locations were counterbalanced across blocks. We separately measured the percentage of "female" responses obtained for each image and fitted a Weibull function to the data using bootstrapping methods (Wichmann \& Hill, 2001a, 2001b) to estimate the baseline PSE threshold.

Adaptation and attentional task. After recording PSEs for male-female discrimination, we determined the contrast-decrement detection threshold, as in Experiment 1. We used the same staircase procedure and criterion of $75 \%$ correct detections. The amount of contrast reduction required ranged from $31 \%$ to $84 \%$. The experimental procedure was the same as in Experiment 1, except for the following differences. Each participant completed two blocks. In the first block, images from the first set of adaptors were presented
Set 1
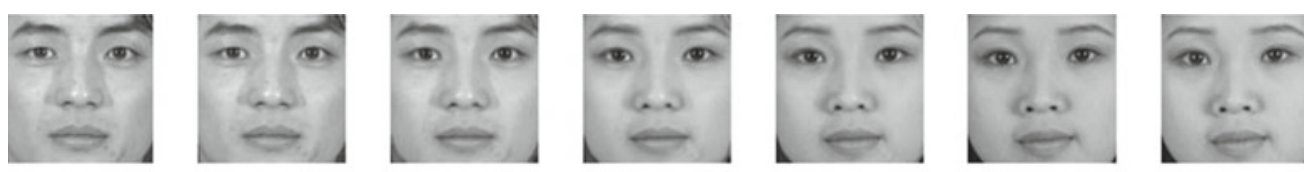

Set 2
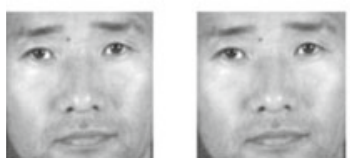

$94 / 6$

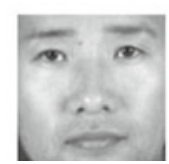

$72 / 28$

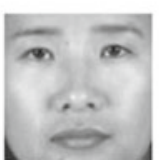

$50 / 50$

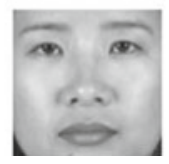

$28 / 72$

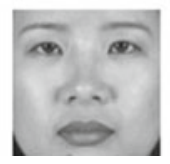

$6 / 94$

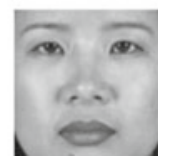

$0 / 100$

Figure 3. Two sets of face stimuli in Experiment 2. Leftmost face is a $100 \%$ male face; rightmost face is $100 \%$ female. Morphed levels were $94 \%-6 \%, 72 \%-28 \%, 50 \%-50 \%, 28 \%-72 \%$, and $6 \%-94 \%$ (male/female); $100 \%$ female faces were used as adaptors. 


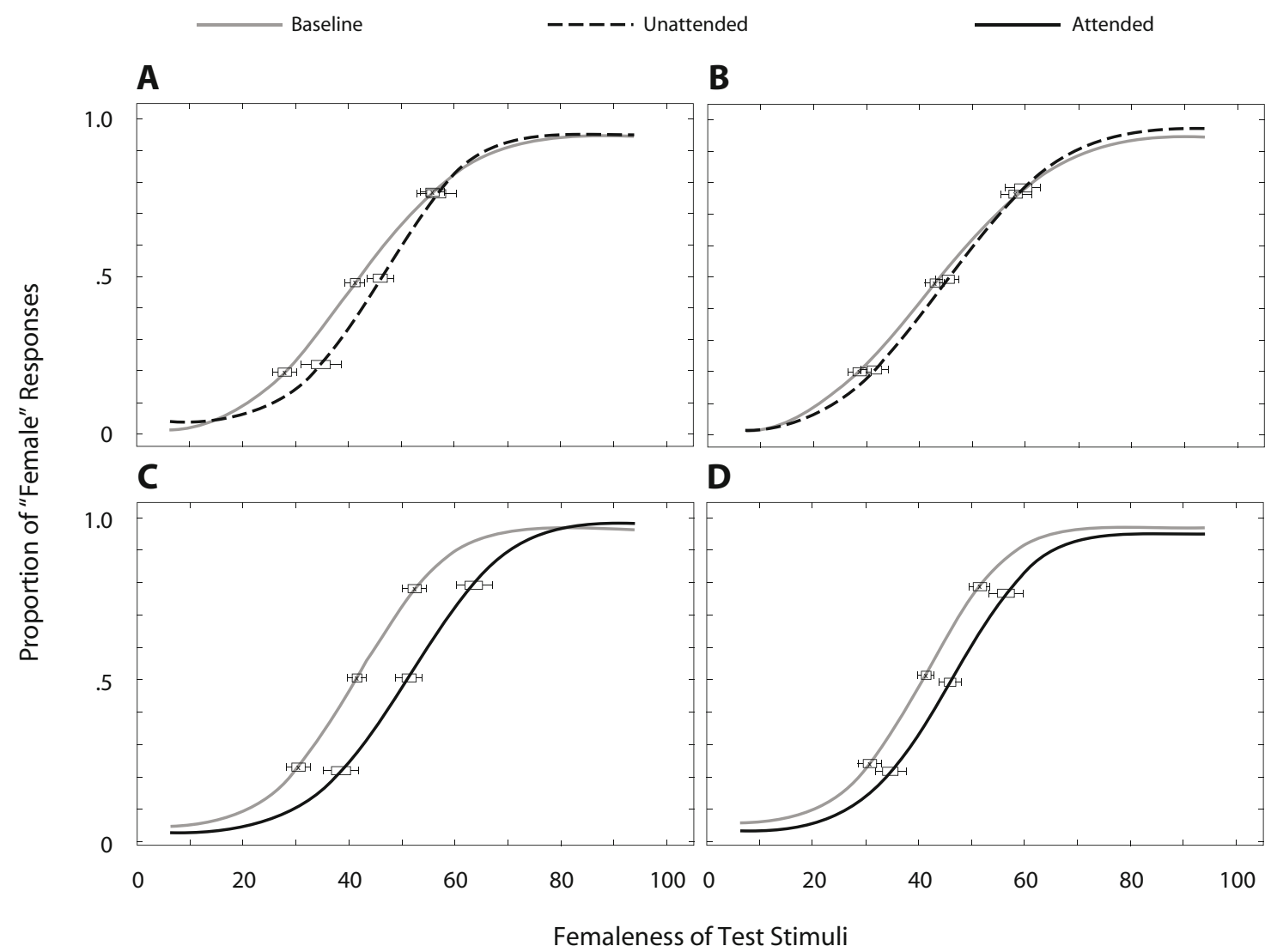

Figure 4. Results of Experiment 2. Each slope was fitted by a Weibull function using the bootstrapping method. (A) Partially suppressed condition for the unattended set. (B) Invisible condition for the unattended set. (C) Partially suppressed condition for the attended set. (D) Invisible condition for the attended set. The error bars indicate a $95 \%$ confidence interval.

to the left visual field, and images from the second set of adaptors to the right visual field. In the second block, the assignment was reversed. Note that we used two different sets of faces for adaptors presented in separate visual fields. We always measured PSEs after adaptation in a different visual field. For example, if the first set was used as an adaptor in the left visual field, the aftereffect of this face was always measured in the right visual field. In this way, we were able to measure high-level adaptation not confined to a specific location (see Moradi et al., 2005).

To replicate the conditions of Moradi et al. (2005), the adaptation period was $4 \mathrm{sec}$, followed by PSE measurement. Throughout the adaptation period, participants performed the contrast-change detection task on the left suppressor and simultaneously tracked their percept, as in Experiment 1. During PSE measurement, a randomly chosen test location (left or right) was cued by flickering, and a test face was presented for $200 \mathrm{msec}$. The test stimuli were always chosen from the same set as the adaptors, but presented in a different location from the adaptor. When participants thought that the face was male, they pressed "1"; otherwise they pressed " 2 ." Each of the two blocks consisted of 150 trials.

\section{Results and Discussion}

We plotted the proportion of "female" responses as a function of femaleness of the stimuli and fitted a Weibull function to the data using the bootstrapping method (Wichmann \& Hill, 2001a, 2001b). PSE was defined as the percentage of femaleness at $50 \%$ "female" responses.
The average PSE before adaptation was 34\% for Set 1 and $56 \%$ for Set 2 in the first block. In the second block, it was $35 \%$ for Set 1 and $46 \%$ for Set 2 . A repeated measures ANOVA revealed that the average PSE of Set $2(51 \%)$ was significantly higher than that of Set $1[35 \% ; F(1,6)=$ $28.311, p<.01]$. Moreover, the interaction between the effect of set and that of block approached significance $[F(1,6)=5.741, p=.054]$. Consequently, we analyzed the two conditions separately.

Figure 4 shows the results of Experiment 2. Like Moradi et al. (2005), we grouped the results according to the reported percept during adaptation into two categories: (1) partially suppressed (the adaptor was visible for more than $1 \mathrm{sec}$ ) and (2) invisible (the adaptor was visible for less than $1 \mathrm{sec})$. The PSE for unattended faces was significantly different, compared with baseline, in the partially suppressed category (Figure 4A), but there was no significant difference for unattended faces in the invisible category (Figure 4B).

The PSE results for attended and unattended faces in the invisible category are shown in Figure 5. To quantify the amount of adaptation, we defined a PSE elevation index (PEI) as the PSE after adaptation divided by that before adaptation, for each set. Note that a PEI value greater than 1 indicates an effect of adaptation. One-sample $t$ tests 


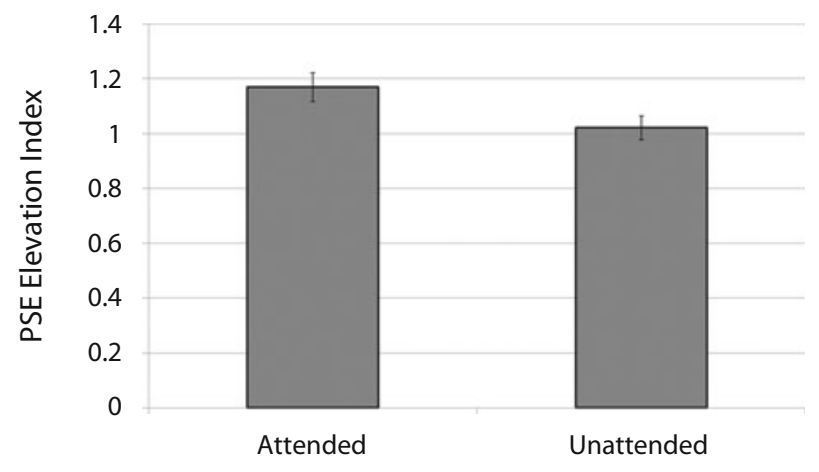

Figure 5. Summary results of Experiment 2. PSE elevation index. Error bars represent standard errors of the means.

indicated that the PEI for the unattended set did not significantly differ from $1[1.02 ; t(6)=0.48, p=.65]$. This suggests that PSEs did not change after adaptation, when participants paid no attention to the location of the invisible adaptor. However, PEI for the attended set significantly differed from $1[1.17 ; t(6)=3.22, p<.05]$, suggesting that significant adaptation occurred for the attended set. Moreover, a paired-sample $t$ test indicated that the PEI of the set in the attended field was significantly higher than that in the unattended field $[t(6)=4.80, p<.01]$, suggesting increased adaptation due to spatial attention.

\section{GENERAL DISCUSSION}

The results above show that endogenous spatial attention is able to influence adaptation to low- as well as to highlevel invisible stimuli. Experiment 1 demonstrated contrastthreshold elevation after adaptation to sinusoidal gratings. Moreover, engaging spatial attention at the location of an invisible adaptor enhanced contrast threshold elevation, compared with a second unattended adaptor location.

The results of Experiment 1 are in line with a recent report by Bahrami et al. (2008). By using a spatial cuing paradigm and low-contrast adaptors, the authors were able to successfully demonstrate the effect of attention on adaptation to line orientations of invisible gratings. However, we believe that our results extend the previous findings by overcoming possible methodological limitations. Bahrami et al.'s paradigm required participants to make a quick response to a target at a previously cued location while adapting to an invisible grating. During the subsequent test phase, a grating was presented at one of the four possible target locations and adaptation was found to be stronger for gratings at the previously attended location than at unattended locations. Although Bahrami and colleagues were able to carefully control attentional engagement during the adaptation phase, residual attentional bias during the test phase may have affected perception of the tilted grating at the attended location; in other words, not controlling the locus of attention during the test period might have allowed remaining spatial attention from the adaptation period to influence the perception of the tilted grating at the previously attended location. It has been demonstrated that sensitivity to low-level contrast gratings is easily enhanced by attention (Ling \& Carrasco, 2006). To control for such an effect, we explicitly cued the spatial location of a test stimulus during the testing phase, effectively resetting attentional locus. Additionally, we equalized individual differences of attentional engagement at the attended location and the amount of adaptation by matching difficulty in the contrast-decrement detection task according to individual thresholds. Finally, we used contrast threshold elevation aftereffects, which presumably occur at earlier stages of processing than do orientation-specific aftereffects (Festman \& Ahissar, 2004), showing that top-down attentional processes may influence the earliest as well as the latest stages of visual processing (see below).

The results of Experiment 2 demonstrate that endogenous spatial attention can influence unconscious face processing. Two recent studies (Finkbeiner \& Palermo, 2009; Moradi et al., 2005) claimed no effect of attention on the processing of invisible faces. Finkbeiner et al. cued spatial attention in a priming paradigm and found that the effect of attention on priming occurred only for nonface stimuli, suggesting a separate route for face-relevant information that is practically immune to endogenous attentional processes. However, the finding may reflect insufficient engagement of attentional resources. A simple cue may fail to capture an adequate amount of attention required to influence high-level unconscious processing. Accordingly, it has been demonstrated that the effect of spatial attention can be significantly increased when one of two stimuli, rather than a single stimulus, is attended (Lavie, 1995; Luck, Chelazzi, Hillyard, \& Desimone, 1997). Furthermore, the contrast-decrement detection task of our study is known to strongly modulate attention (Braun, 1994; Zenger-Landolt \& Heeger, 2003). The same argument can be made for Moradi et al.'s study, where participants were simply instructed to attend to a moving suppressor. This possibility is supported by the fact that some aspects of face processing - for example, emotional expressions (Pessoa et al., 2002) - are influenced by high attentional load. Note that Finkbeiner and Palermo used a gender discrimination task to measure the effect of masked priming, and Moradi et al. used a face identification task to measure the effects of adaptation. It is possible that these two studies did not find an effect of attention on invisible stimuli because of these task differences from our study. However, since gender and identity discrimination of faces are based on similar features, such as the regions around the eyes and mouth (Mangini \& Biederman, 2004), we think that the different results are unlikely to be due to differences in the task and probably stem from insufficient engagement of attention in the previous studies.

The results of Experiment 2 show a significant gender aftereffect for face stimuli suppressed from awareness. Importantly, we used the same adaptation paradigm as for the low-level stimuli in Experiment 1, including the same task at the attended location. To the best of our knowledge, this is the first time an attentional effect on unconscious processing of low- and high-level stimuli has been re- 
corded using the same paradigm. The results indicate that attention acts at multiple stages of the visual hierarchy, independently of awareness. Although for high-level stimuli depth of suppression under binocular rivalry may usually not allow adaptation (Nguyen, Freeman, \& Alais, 2003), attention may have effectively strengthened the adaptor (Boynton \& Finney, 2003), producing a significant aftereffect. Therefore, the role of attention in strengthening perceptual processes (Boynton, 2004; Pestilli, Viera, \& Carrasco, 2007) may extend to the realm of unconscious processing.

In summary, we found that spatial attention influenced the amount of low- as well as high-level adaptation to invisible stimuli, suggesting a dissociation between the neural mechanisms underlying attention and visual awareness.

\section{AUTHOR NOTE}

This work was supported by the Korea Science and Engineering Foundation (KOSEF) Grant R01-2008-000-10820-0 funded by the Korean government (MEST). The first two authors contributed equally to this work. Correspondence concerning this article should be addressed to S. C. Chong, Department of Psychology, Yonsei University, 262 Seongsangno, Seodaemun-gu, Seoul, Korea 120-749 (e-mail: scchong@ yonsei.ac.kr).

\section{REFERENCES}

Bahrami, B., Carmel, D., Walsh, V., Rees, G., \& Lavie, N. (2008). Spatial attention can modulate unconscious orientation processing. Perception, 37, 1520-1528. doi:10.1167/8.3.12

BLAKE, R. (2001). A primer on binocular rivalry, including current controversies. Brain \& Mind, 2, 5-38.

Blake, R., Tadin, D., Sobel, K. V., Raissian, T. A., \& Chong, S. C. (2006). Strength of early visual adaptation depends on visual awareness. Proceedings of the National Academy of Sciences, 103, 47834788. doi:10.1073/pnas.0509634103

Boynton, G. M. (2004). Adaptation and attentional selection. Nature Neuroscience, 7, 8-10. doi:10.1038/nn0104-8

Boynton, G. M., \& Finney, E. M. (2003). Orientation-specific adaptation in human visual cortex. Journal of Neuroscience, 23, 8781-8787.

Brainard, D. H. (1997). The Psychophysics Toolbox. Spatial Vision, 10, 433-436.

BRAUN, J. (1994). Visual search among items of different salience: Removal of visual attention mimics a lesion in extrastriate area V4. Journal of Neuroscience, 14, 554-567.

Chung, C., OH, K., Lee, Y., \& Byun, H. (1998). Development of a "Gamsung" measurement system and a database for facial expressions and gestures (Tech. Rep. No. G-17-01-09). Korea: Ministry of Science and Technology.

Festman, Y., \& Ahissar, M. (2004). Attentional states and the degree of visual adaptation to gratings. Neural Networks, 17, 849-860. doi:10.1016/j.neunet.2004.02.006

Finkbeiner, M., \& Palermo, R. (2009). The role of spatial attention in nonconscious processing: A comparison of face and nonface stimuli. Psychological Science, 20, 42-51. doi:10.1111/j.1467 $-9280.2008 .02256 . \mathrm{x}$

He, S., Cavanagh, P., \& Intriligator, J. (1996). Attentional resolution and the locus of visual awareness. Nature, 383, 334-337.
Hochstein, S., \& Ahissar, M. (2002). View from the top: Hierarchies and reverse hierarchies in the visual system. Neuron, 36, 791-804.

JiANG, Y., \& HE, S. (2006). Cortical responses to invisible faces: Dissociating subsystems for facial-information processing. Current Biology, 16, 2023-2029. doi:10.1016/j.cub.2006.08.084

Kanai, R., Tsuchiya, N., \& Verstraten, F. A. J. (2006). The scope and limits of top-down attention in unconscious visual processing. Current Biology, 16, 2332-2336. doi:10.1016/j.cub.2006.10.001

KASTNER, S., \& UNGERLEIDER, L. [G.] (2000). Mechanisms of visual attention in the human cortex. Annual Review of Neuroscience, 23, 315-341.

LAVIE, N. (1995). Perceptual load as a necessary condition for selective attention. Journal of Experimental Psychology: Human Perception \& Performance, 21, 451-468.

LevitT, H. (1970). Transformed up-down methods in psychoacoustics. Journal of the Acoustical Society of America, 49, 467-477.

Ling, S., \& Carrasco, M. (2006). Sustained and transient covert attention enhance the signal via different contrast response functions. Vision Research, 46, 1210-1220. doi:10.1016/j.visres.2005.05.008

Luck, S. J., Chelazzi, L., Hillyard, S. A., \& Desimone, R. (1997). Neural mechanisms of spatial selective attention in areas V1, V2, and V4 of macaque visual cortex. Journal of Neurophysiology, 77, 24-42.

Mangini, M. C., Biederman, I. (2004). Making the ineffable explicit: Estimating the information employed for face classifications. Cognitive Science, 28, 209-226. doi:10.1016/j.cogsci.2003.11.004

MARR, D. (1982). Vision: A computational investigation into the human representation and processing of visual information. San Francisco: Freeman.

Moradi, F., Koch, C., \& Shimojo, S. (2005). Face adaptation depends on seeing the face. Neuron, 45, 169-175.

Nguyen, V. A., Freeman, A. W., \& Alais, D. (2003). Increasing depth of binocular rivalry suppression along two visual pathways. Vision Research, 43, 2003-2008. doi:10.1016/S0042-6989(03)00314-6

Pelli, D. G. (1997). The VideoToolbox software for visual psychophysics: Transforming numbers into movies. Spatial Vision, 10, 437-442.

Pessoa, L., McKenna, M., Gutierrez, E., \& Ungerleider, L. G. (2002). Neural processing of emotional faces requires attention. Proceedings of the National Academy of Sciences, 99, 11458-11463. doi:10.1073/pnas.172403899

Pestilli, F., Viera, G., \& Carrasco, M. (2007). How do attention and adaptation affect contrast sensitivity? Journal of Vision, 7, 1-12. doi:10.1167/7.7.9

Reynolds, J. H., \& Desimone, R. (1999). The role of neural mechanisms of attention in solving the binding problem. Neuron, 24, 19-29.

TyLER, C. W. (1997). Colour bit-stealing to enhance the luminance resolution of digital displays on a single pixel basis. Spatial Vision, 10, 369-377.

Watson, A. B., \& Pelli, D. G. (1983). QUEST: A Bayesian adaptive psychometric method. Perception \& Psychophysics, 33, 113-120.

Wichmann, F. A., \& Hill, N. J. (2001a). The psychometric function: I. Fitting, sampling, and goodness of fit. Perception \& Psychophysics, 63, 1293-1313.

WichmanN, F. A., \& Hill, N. J. (2001b). The psychometric function: II. Bootstrap-based confidence intervals and sampling. Perception \& Psychophysics, 63, 1314-1329.

Zenger-Landolt, B., \& HeEger, D. J. (2003). Response suppression in V1 agrees with psychophysics of surround masking. Journal of Neuroscience, 23, 6884-6893.

(Manuscript received March 3, 2009; revision accepted for publication May 18, 2009.) 